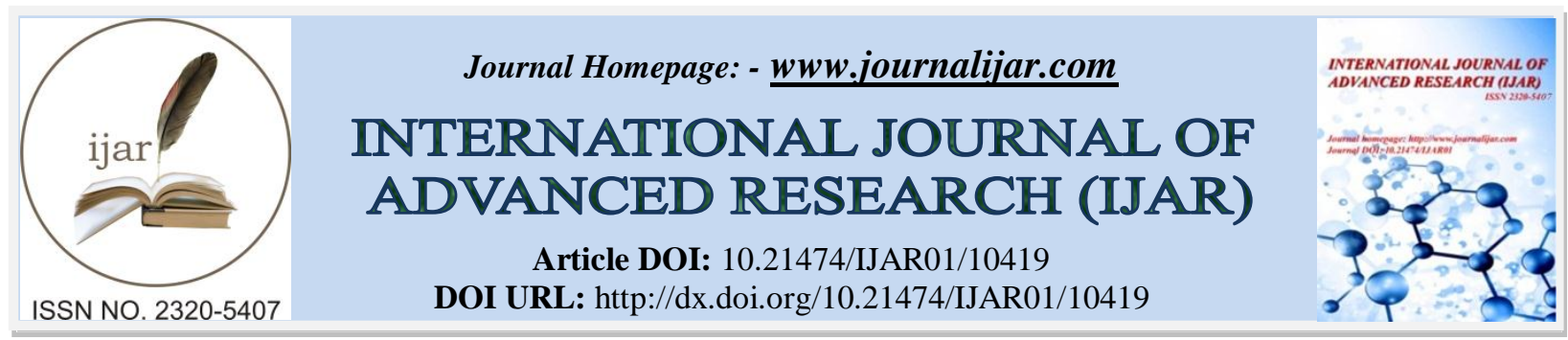

RESEARCH ARTICLE

\title{
PULMONARY ASPERGILLOSIS COMPLICATED WITH HAEMOPHAGOCYTIC SYNDROME SECONDARY TO CHRONIC GRANULOMATOUS DISEASE DESCRIPTION OF THE FIRST CASE
}

\section{Dr. Luis Andrés Dulcey ${ }^{1}$, Jonathan Antonio Pineda ${ }^{2}$, Jose Jorge Sampay ${ }^{2}$, Hector Alonso Moreno ${ }^{2}$ and Raimondo Caltagirone ${ }^{3}$}

1. Hospital Universitario de los Andes Nivel Mezanina Servicio de Medicina Interna. Mérida Venezuela. Correo.

2. Especialista en Medicina Interna. Facultad de Medicina. Universidad de los Andes. Mérida. Venezuela.

3. Médico Internista e Intensivista Facultad de Medicina. Universidad de los Andes. Mérida.Venezuela.

\section{Manuscript Info}

.........................

Manuscript History

Received: 30 November 2019

Final Accepted: 31 December 2019

Published: January 2020

Key words:-

Invasive Pulmonary Aspergillosis, Lymphohistiocytosis Hemophagocytic, Granulomatous Disease, Chronic, Immunosuppression

\section{Abstract}

Chronic granulomatous disease is a primary immunodeficiency caused by mutations in the genes of the 4 subunits of NADPH oxidase. Among the clinical findings are lymphadenopathies, hepatosplenomegalies and recurrent pneumonia. It is a 16-year-old male who presents productive cough with purulent expectoration, as well as progressive dyspnea and a loss of about 10 kilos of weight in the last 3 months, at the cervical level it presents bilateral adenomegalies, cardiopulmonary rhonchi scattered without cardiac alterations, at an abdominal hepatosplenomegaly, pancytopenic is found in the admission and an image compatible with an aspergilloma is evident in the thoracic radiograph. Bone marrow aspirate was performed with positive culture for fungi compatible with Aspergillus, staining and flow cytometry were performed with confirmation of Hemophagocytosis and Chronic Granulomatous Disease. This case is exceptional because it is the first report where the 3 entities coexist in the same patient.

Copy Right, IJAR, 2020,. All rights reserved.

\section{Introduction:-}

La enfermedad granulomatosa crónica (EGC) considerada como una inmunodeficiencia primaria es causada por mutaciones en los genes que codifican para cualquiera de las 4 subunidades que conforman a la enzima NADPH oxidasa, encargada de regular la producción de especies oxidantes microbicidas que constituyen la primera barrera de defensa del ser humano contra los microorganismos infecciosos. Esta es una deficiencia muy heterogénea clasificada en (EGC) ligada al cromosoma x (subunidad gp91-phox) y autosómica recesiva ${ }^{1-2}$. La (EGC) se clasifica según el modo de herencia en: herencia recesiva ligada al cromosoma x y herencia autosómica recesiva.

Dentro de los hallazgos clínicos más frecuentes se encuentran entre otros las adenopatías, hepatoesplenomegalias y neumonías a repetición ${ }^{3-4}$. Esta enfermedad se caracteriza por infecciones recurrentes causadas por gérmenes piógenos catalasa positivas, y otros patógenos o de baja virulencia. Entre los más frecuentes se encuentran staphylococcus aureus, echerichia coli, pseudomona aeruginosa, salmonella, nocardia, serratia, etc y hongos ${ }^{5}$, dentro de los cuales es el aspergillus el mayor responsable de mortalidad ${ }^{6}$.

En cuanto a la epidemiologia de la Apergilosis pulmonaresta depende de múltiples factores de riesgo ${ }^{7}$ como lo han demostrado algunos estudios. Por su baja frecuencia las descripciones sobre aspergilosis pulmonar complicada con síndrome hemofagocitico están descritas en la literatura médica principalmente en el campo de las neoplasias 
hematológicas ${ }^{8}$, dichas descripciones son muy escasas y másaún en el contexto de enfermedad granulomatosa crónica. El objetivo principal del presente reporte de caso es dar a conocer nuestra experiencia como unidad de Medicina Interna, en la atención de este paciente en quien se reporta por primera vez la coexistencia de 3 entidades cada una de ellas menos frecuente que la anterior.

\section{Presentación del Caso:-}

Motivo de consulta:

Disnea.

\section{Enfermedad actual:}

Se trata de paciente masculino de 16 años de edad natural y procedente de Lagunillas Mérida es traído por familiar por presentar inicio de enfermedad actual a mediados de mayo de 2017, caracterizado por alzas térmicas no cuantificadas a predominio vespertino así como pérdida de unos 10 kilos de peso en 2 meses, el día 28 de junio de 2017 se asocia tos productiva con expectoración mucopurulenta así como disnea que progresa de moderados a pequeños esfuerzos motivo por el cual es traído a este centro para valoración donde posterior a ella se decide su ingreso en el mes de julio de 2017.

\section{Antecedentes:-}

Comorbilidades: niega cardiometabolicas, niega neurológicas, niega respiratorias crónicas. Familiares padres vivos aparentemente sanos, de importancia ambos primos maternos, resto hermanos aparentemente sanos. Religión testigo de Jehová. Psicobiologicos niega oh u chimoico.Epidemiológicos: Tuberculosis ganglionar a los 9 años de edad. Inicio el 18 de enero de 2010 rifampicina 600 isoniacida 600 y pirazinamida $750 \mathrm{mg}$ día posterior a biopsia ganglionar que revelo Bacilos acido alcohol resistentes, recibe terapéutica durante 6 meses.

En enero de 2011 presenta diagnóstico de Tuberculosis pulmonar por hallazgo imagenologico con Baciloscopias negativas realizándose fibrobroncoscopia con cepillado y lavado bronco alveolar, así como reacción en cadena de polimerasa de alta sensibilidad para micobacterias la cual demostró la presencia del germen, inicia tratamiento el 10 de octubre de 2011, recibe ethambutol adicional a los medicamentos que recibió en 2010.

\section{Examen Funcional y Físico:-}

Refiere disnea que progresa de moderados a pequeños esfuerzos, niega dolor torácico, tolera el decúbito. No nuevas alzas térmicas.Refiere haber acudido en múltiples ocasiones al centro de salud de su localidad.

Signos vitales TA: 93/44 FC: 104 FR: 28 Sat 02 92\% Fio2 30\%. Peso 40 kilos talla $152 \mathrm{~cm}$. Cabeza normocefalo sin alteraciones. Otorrinolaringologico mucosa oral seca resto sin alteración. Cuello móvil no bocio sin signos de meningismo ni presencia de signos de congestión. Adenomegalias en segmentos laterocervicales no adheridos de consistencia blanda. Tórax taquipneico con tirajes murmullo presente con múltiples roncus a la auscultación. Ruidos cardiacos sin soplo y sin galope. Abdomen con visceromegalias hepatoesplenomegalia hepatometria 10 × 12 × 14 $\mathrm{cm}$ Boyd II, ruidos intestinales presentes. Extremidades eutróficas simétricas sin edema. Neurológico Glasgow 15 vigil activo orientado. Funciones mentales superiores lenguaje sin alteración. Fuerza muscular V de V. Reflejos conservados. Pares craneales no evidencian alteración.

Exámenes paraclínicos realizados:-

Tabla 1:- Exámenes paraclínicos de Ingreso.

\begin{tabular}{|l|l|}
\hline Examen & Valores \\
\hline Hematología & Hemoglobina 7.6 gramos Hcto 23 VCM 77.5 HCM 24 \\
& $\begin{array}{l}\text { leucocitos 2000 células segmentados 61\% Linfocitos } \\
30 \% \text { Valor absoluto de Neutrofilos de 1220 células } \\
\text { Plaquetas 126000. }\end{array}$ \\
\hline Uroanalisis & $\begin{array}{l}\text { Densidad } 1020 \text { No cilindros No presencia de eritrocitos, } \\
\text { Piocitos Negativos }\end{array}$ \\
\hline Creatinina & $0,6 \mathrm{mg} / \mathrm{dL}$ \\
\hline Urea & $25 \mathrm{mg} / \mathrm{dL}$ \\
\hline Glicemia & $98 \mathrm{mg} / \mathrm{dL}$ \\
\hline
\end{tabular}




\begin{tabular}{|l|l|}
\hline TGO y TGP & 24 y 18 UI \\
\hline Proteínas totales & $\begin{array}{l}6 \text { gramos (Albumina 3,4 gramos) (Globulinas 2,6 } \\
\text { gramos) }\end{array}$ \\
\hline Serologia de 4ta Generación para VIH & Negativa \\
\hline
\end{tabular}

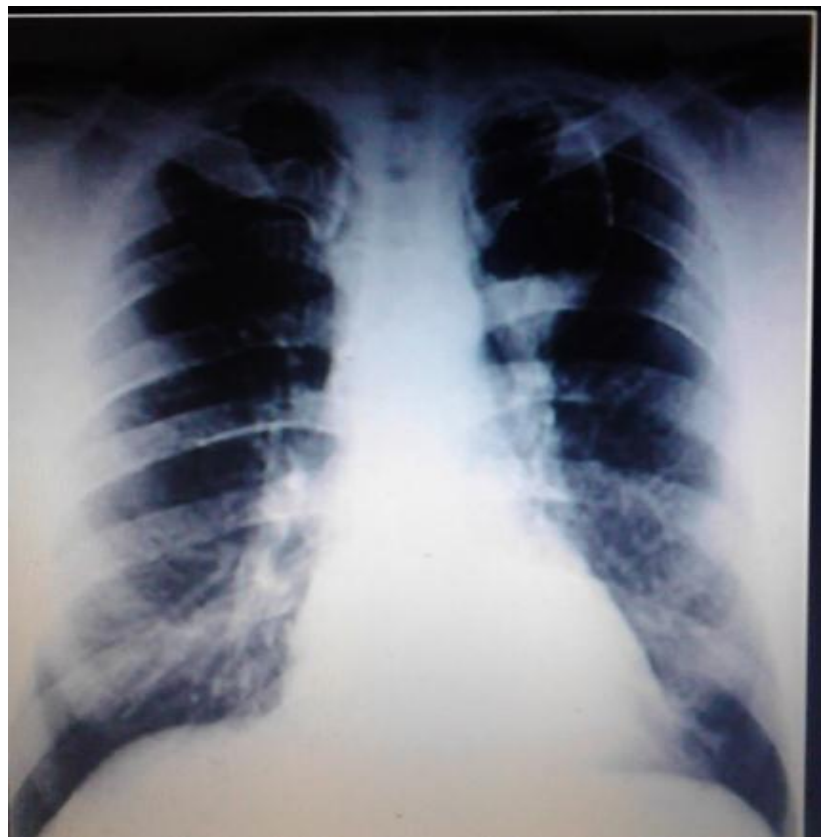

Imagen 1:- Radiografía PA de Tórax. RX de tórax muestra una neumonía compatible con aspergiloma en campo pulmonar superior izquierdo.

El Eco abdominal muestra una hepatoesplenomegalia sin signos de hipertensión portal. Se realizan 3 baciloscopias todas siendo negativas. La serología para micosis profundas realizada en Julio de 2017 fue positiva para aspergillus. Se ordenan niveles de ferritina los cuales fueron mayores a $1200 \mathrm{mcg}$ y niveles de triglicéridos en $360 \mathrm{mg} / \mathrm{dl}$. Los niveles de fibrinógeno se encontraban normales. En base a lo dispuesto se interconsulta a Hematología quienes deciden realizar un aspirado de medula ósea por la pancitopenia. Los hallazgos muestran un aumento de los sideroblastos en anillo con tinción de azul de Prusia positivo e importante compromiso de todas las líneas celulares con hemofagocitos presentes. Se realiza un mielocultivo siendo positivo para hongos compatible con aspergillus. En ese momento se realiza el planteamiento de un síndrome hemofagocitico secundario a infección por aspergillus.

Tabla 2:- Criterios diagnósticos Síndrome Hemofagocitico ${ }^{8}$.

Fiebre mayor a 38 grados por 7 días

Hepatomegalia o esplenomegalia

Citopenia de 2 o más líneas celulares

Hipertrigliceridemia o hipofibrinogenemia

Hemofagocitosis en medula ósea

Ferritina mayor a 500 ug

Receptor CD25 positivo

Actividad baja de células NK

Se decidió realizar una biopsia de ganglio linfático cervical, dicha muestra es llevada a patología donde se ordenó adicional a la biopsia tinciones para agentes micoticos y micobacterianos, siendo positiva para el primero observándose hifas compatibles con aspergillus. Nos planteamos la posibilidad de una inmunodeficiencia primaria, por lo que se ordenaron niveles de inmunoglobulinas los cuales se encontraban dentro de la normalidad.El recuento de células CD4 y CD8 ${ }^{9}$ eran normales, pero los marcadores para células NK se encontraban bajos ${ }^{10-11}$. El marcador CD25 resulto ser positivo ${ }^{12}$. Ante dichos hallazgos se ordenó realizar prueba de nitroazul de tetrazolio el cual mostro una disminución de la actividad de la NADPH ${ }^{13-14}$. Estas últimas pruebas fueron realizadas por citometria de flujo. 
Se decidió mantener la indicación de anfotericina hasta alcanzar una dosis acumulada de 1.5 gramos. El paciente a los 25 días del mes de julio de 2017 presenta un empeoramiento de su condición general, requirió ventilación mecánica invasiva y tras encontrarse 48 horas conectado a esta, el referido paciente fallece producto de una falla multiorganica.

\section{Discusión:-}

La aspergilosis pulmonar es una infección infrecuente en pacientes inmunocompetentes, siendo más frecuente en inmunocomprometidos ${ }^{15-16}$, mucho más aún lo es el síndrome hemofagocitico asociado a dicha infección ${ }^{17}$. Existen reportes muy escasos de asociación entre la presencia de aspergilosis pulmonar complicada con síndrome hemofagocitico, sobre todo en pacientes con condiciones de inmunosupresión como en el caso de neoplasias hematológicas ${ }^{18-19}$, en dichos casos la terapéutica está orientada a tratar la enfermedad de base ${ }^{20-21}$.

La terapéutica de los pacientes con Aspergilosis está orientada a manejarse con fármacos como la Anfotericina B en cualquiera de sus formas ${ }^{22}$, a largo plazo debe mantenerse fármacos como el Voriconazol o Posaconazol, en el presente caso se indicó Anfotericina B no lográndose iniciar terapia de mantenimiento por el fallecimiento del paciente. Dentro de los pilares fundamentales se encuentra la prevención y administración de vacunas ${ }^{23}$.

\section{Conclusiones:-}

Las inmunodeficiencias primarias son una causa frecuente de infecciones por gérmenes atípicos, debemos considerar en todo paciente que presente de forma recurrente infecciones por agentes oportunistas la posibilidad de que exista dicha asociación ${ }^{24}$. El diagnóstico es impreciso en la mayoría de los casos y requiere de una experticia por parte de personal médico entrenado en este tipo de pacientes. Se espera que en un futuro próximo la terapia génica sea una alternativa terapéutica distinta al trasplante de medula ósea ${ }^{25}$.

\section{Referencias:-}

1. Roos D, de Boer M. Retrotransposable genetic elements causing neutrophil defects. Eur J Clin Invest. 2018 May 17:e12953. doi: 10.1111/eci.12953.

2. Slack MA, Thomsen IP. Prevention of Infectious Complications in Patients With Chronic Granulomatous Disease. J Pediatric Infect Dis Soc. 2018 May 9;7(suppl_1):S25-S30. doi: 10.1093/jpids/piy016

3. Connelly JA, Marsh R, Parikh S, Talano JA. Allogeneic Hematopoietic Cell Transplantation for Chronic Granulomatous Disease: Controversies and State of the Art. J Pediatric Infect Dis Soc. 2018 May 9;7(suppl_1):S31-S39. doi: 10.1093/jpids/piy015

4. Henrickson SE, Jongco AM, Thomsen KF, Garabedian EK, Thomsen IP. Noninfectious Manifestations and Complications of Chronic Granulomatous Disease. J Pediatric Infect Dis Soc. 2018 May 9;7(suppl_1):S18-S24. doi: 10.1093/jpids/piy014.

5. Rider NL, Jameson MB, Creech CB. Chronic Granulomatous Disease: Epidemiology, Pathophysiology, and Genetic Basis of Disease. J Pediatric Infect Dis Soc. 2018 May 9;7(suppl_1):S2-S5. doi: 10.1093/jpids/piy008.

6. Bennett N, Maglione PJ, Wright BL, Zerbe C. Infectious Complications in Patients With Chronic Granulomatous Disease. J Pediatric Infect Dis Soc. 2018 May 9;7(suppl_1):S12-S17. doi: 10.1093/jpids/piy013.

7. Kalua K, Zimba B, Denning DW. Estimated Burden of Serious Fungal Infections in Malawi. J Fungi (Basel). 2018 May 21;4(2). pii: E61. doi: 10.3390/jof4020061.

8. van de Peppel RJ, Visser LG, Dekkers OM, de Boer MGJ. The burden of Invasive Aspergillosis in patients with haematological malignancy: A meta-analysis and systematic review. J Infect. 2018 Jun;76(6):550-562. doi: 10.1016/j.jinf.2018.02.012.

9. Lee JY, Kim JH, Lee JS, Ryu JM, Seo JJ, Im HJ. Initial Characteristics and Clinical Severity of Hemophagocytic Lymphohistiocytosis in Pediatric Patients Admitted in the Emergency Department. Pediatr Emerg Care. 2018 May 23. doi: 10.1097/PEC.0000000000001518.

10. An Q, Wang Y, Hu S, Fang D, Xuan C, Xu S, Jin M, Ji Q. Clinical significance of lymphocyte subset changes in hemophagocytic lymphohistiocytosis of children.Exp Ther Med. 2016 Dec;12(6):3549-3552. doi: 10.3892/etm.2016.3809.

11. Al-Samkari H, Berliner N. Hemophagocytic Lymphohistiocytosis.Annu Rev Pathol. 2018 Jan 24;13:27-49. doi: 10.1146/annurev-pathol-020117-043625.

12. Ochoa S, Cheng K, Fleury CM, Luccioli S, Bellanti JA. A 28-year-old woman with fever, rash, and pancytopenia. Allergy Asthma Proc. 2017 Jul 1;38(4):322-327. doi: 10.2500/aap.2017.38.4042. 
13. Wang Y, Liu D, Zhu G, Yin C, Sheng G, Zhao X. [Significance of soluble CD163 and soluble CD25 in diagnosis and treatment of children with hemophagocytic lymphohistiocytosis]. Zhonghua Er Ke Za Zhi. 2015 Nov;53(11):824-9. Chinese

14. Kulkarni M, Desai M, Gupta M, Dalvi A, Taur P, Terrance A, Bhat S, Manglani M, Raj R, Shah I, Madkaikar M. Clinical, Immunological, and Molecular Findings of Patients with p47<sup $>$ phox $</$ sup $>$ Defect Chronic Granulomatous Disease (CGD) in Indian Families. J Clin Immunol. 2016 Nov;36(8):774-784.

15. Iacobini M, Duse M, Di Coste A, Balducci L. Improvement of a rapid screening test for chronic granulomatous disease. Int J Immunopathol Pharmacol. 2013 Jul-Sep;26(3):807-8.

16. Loeffert ST, Hénaff L, Dupont D, Bienvenu AL, Dananché C, Cassier P, Bénet T, Wallon M, Gustin MP, Vanhems P. Prospective survey of azole drug resistance among environmental and clinical isolates of Aspergillus fumigatus in a French University hospital during major demolition works. J Mycol Med. 2018 May 28. pii: S1156-5233(17)30447-X. doi: 10.1016/j.mycmed.2018.05.007.

17. Fernandes J, Hamidi F, Beau R, Leborgne R, Castier Y, Mordant P, Boukkerou A, Latgé JP, Pretolani M. Penetration of the Human Pulmonary Epithelium by Aspergillus fumigatus Hyphae. J Infect Dis. 2018 May 26. doi: 10.1093/infdis/jiy298.

18. Majima S, Okachi S, Asano M, Wakahara K, Hashimoto N, Sato M, Ishigami M, Hasegawa Y. Pseudomembranous Invasive Tracheobronchial Aspergillosis with Fulminant Hepatitis and Hemophagocytic Syndrome. Intern Med. 2018 Mar 30. doi: 10.2169/internalmedicine.9673-17.

19. Yamada A, Moritake H, Sawa D, Shimonodan H, Kojima H, Kamimura S, Nunoi H. [Refractory acute myeloid leukemia developed malignancy-associated hemophagocyticlymphohistiocytosis during treatment of invasive fungal infection]. RinshoKetsueki. 2013 Apr;54(4):383-7.

20. Foley JM, Borders H, Kurt BA. A Diagnostic Dilemma: Similarity of Neuroradiological Findings in Central Nervous System HemophagocyticLymphohistiocytosis and Aspergillosis. PediatrBloodCancer. 2016 Jul;63(7):1296-9. doi: 10.1002/pbc.25967.

21. Calmettes C, Gabriel F, Blanchard E, Servant V, Bouchet S, Kabore N, Forcade E, Leroyer C, Bidet A, Latrabe V, Leguay T, Vigouroux S, Tabrizi R, Breilh D, Accoceberry I, de Lara MT, Pigneux A, Milpied N, Dumas PY. Breakthrough invasive aspergillosis and diagnostic accuracy of serum galactomannan enzyme immune assay during acute myeloid leukemia induction chemotherapy with posaconazole prophylaxis. Oncotarget. 2018 Jun 1;9(42):26724-26736. doi: 10.18632/oncotarget.25477.

22. Bagshaw E, Enoch DA, Blackney M, Posthumus J, Kuessner D. Economic impact of treating invasive mold disease with isavuconazole compared with liposomal amphotericin B in the UK. FutureMicrobiol. 2018 Jun 18. doi: 10.2217/fmb-2018-0119.

23. Comité Nacional de Infectología; Grupo de Trabajo de Inmunología. Consensus on updating immunizations in patients with primary immunodeficiencies. Arch Argent Pediatr. 2018 Apr 1;116(2):s20-s33. doi: 10.5546/aap.2018.s20.

24. Duarte Ferreira R, Silva S, Carrapatoso I, Regateiro F, Santos N, Silva D, Sousa F, Oliveira S, Paiva M, Torres da Costa J, Câmara R, Faria E, Lopes da Silva S; Grupo de Interesse de Imunodeficiências Da Sociedade Portuguesa de Alergologia e Imunologia Clínica. [Letter to the Editor: Primary Immunodeficiencies in Adults Multicentric Cooperation to Characterize the Portuguese Reality]. Acta Med Port. 2018 Apr 30;31(4):231-232. doi: 10.20344/amp.10572.

25. Keller MD, Notarangelo LD, Malech HL. Future of Care for Patients With Chronic Granulomatous Disease: Gene Therapy and Targeted Molecular Medicine.J Pediatric Infect Dis Soc. 2018 May 9;7(suppl_1):S40-S44. doi: 10.1093/jpids/piy011. 